\title{
Monocenter feasibility study of the MRI compatibility of the Evia pacemaker in combination with Safio S pacemaker lead
}

\author{
Christian G Wollmann ${ }^{1,3^{*}}$, Erich Steiner ${ }^{2}$, Paul Vock ${ }^{1,3}$, Bonaventure Ndikung ${ }^{4}$ and Harald Mayr ${ }^{1,3}$
}

\begin{abstract}
Background: The purpose of this study was to evaluate the feasibility of the magnetic resonance (MR) conditional pacemaker (PM) system (Evia SR-T and DR-T with Safio S leads) under MR conditions.

Methods: Patients with standard PM indications and Evia PM were eligible for enrollment in this single center prospective non-randomized pilot study. Patients underwent MR of the brain and lower lumbar spine at 1.5 Tesla. Atrial (RA) und ventricular (RV) lead parameters (sensing, pacing threshold [PTH], pacing impedance) were assessed immediately before (baseline follow-up [FU]) and immediately after MRI ( $\left.1^{\text {st }} \mathrm{FU}\right)$, after 1 month $\left(2^{\text {nd }} \mathrm{FU}\right)$ and 3 months ( $\left.3^{\text {rd }} \mathrm{FU}\right)$. The effect of MR on serious adverse device effect (SADE) free-rate, on atrial and ventricular sensing (ASNS; mV) and atrial (RA) and ventricular (RV) pacing thresholds (PTH; V/0.4 ms) were investigated between baseline and $2^{\text {nd }} \mathrm{FU}$. Continuous variables are expressed as mean $\pm S D$ and were compared using paired Student's t-test. A $p<0.05$ was considered significant.

Results: Thirty-one patients were enrolled. One patient had to be excluded because of an enrollment violation. Therefore, data of 30 patients (female 12 [40\%], age $73 \pm 12$ years, dual chamber PM 15 [50\%]) were included in this analysis. No MR related SADE occurred. Lead measurements were not statistically different between the baseline FU and the $2^{\text {nd }} F U$ (ASNS at baseline 3.2 $\pm 2.1 / 15.0 \pm 6.0$, at $2^{\text {nd }} F U 3.2 \pm 2.1 / 14.9 \pm 6.5 ; p=n s$. RA-PTH/RV-PTH at baseline $0.68 \pm 0.18 / 0.78 \pm 0.22$, at $\left.2^{\text {nd }} F U 0.71 \pm 0.24 / 0.78 \pm 0.22 ; p=n s\right)$. The presence of the permanent pacemakers led to MR imaging artifacts on diffusion weighted sequences of the brain, but did not affect other sequences (e.g. FLAIR and T2 weighted spin-echo images).
\end{abstract}

Conclusion: The use of the MR conditional Evia PM in a MR environment under predefined conditions is feasible. No MR related SADEs nor clinically relevant changes in device functions occurred.

Keywords: Pacemaker, Magnetic resonance, Adverse events, Follow-up, Remote monitoring

\section{Background}

Magnetic resonance imaging (MRI) has become an important tool for diagnosis and therapy control of many diseases [1]. Patients with cardiac implantable electronic devices such as permanent pacemakers and implantable defibrillators are usually excluded from MRI examinations since the majority of implantable devices have a contraindication for MRI $[2,3]$. Some larger series with pacemaker patients who underwent MR suggest an

\footnotetext{
* Correspondence: Christian.wollmann@stpoelten.lknoe.at

'Hospital of St. Pölten-Lilienfeld, Department of Cardiology, St. Pölten, Austria ${ }^{3}$ Karl Landsteiner Society, Institute of Research on Ischemic Heart Diseases and Rhythmology, St. Pölten, Austria

Full list of author information is available at the end of the article
}

acceptable risk-benefit ratio by taking MRI and pacemaker related precautions [4-7]. Potential (adverse) effects of MR on pacemaker function include effects from different sources $[2,7]$. The static magnetic field may induce mechanical forces on ferromagnetic components, unpredictable magnetic sensor activation, reedswitch closure and changes in electrocardiograms. Potential effects from modulated radio frequency (RF) fields are heating of cardiac tissue adjacent to lead electrodes potentially leading to pacing threshold increases, possible induction of arrhythmias, pacemaker reprogramming or reset, RF interactions with the device (over-and undersensing). Gradient magnetic fields may

\section{Biomed Central}


induce arrhythmias, and voltages on leads causing overand under-sensing. Combined field effects are alterations of device function because of electro-magnetic interference, mechanical forces (vibration), electrical reset of devices, and damage to pacemaker and/or leads.

As a result of patients' needs (and their physicians') different manufacturers recently market released permanent implantable pacemaker systems which are designed with respect to the above mentioned potential adverse effects of MR on permanent pacemakers. The first of these especially designed pacemaker systems has been proven in a prospective, randomized study published showing the safety of a MR conditional dual chamber pacemaker system [8].

By mid of 2010 the MR conditional EVIA pacemaker systems (Biotronik SE \& Co KG, Berlin, Germany) became available in Europe, offering the Evia pacemaker family [Evia $\mathrm{SR}(-\mathrm{T})$ and $\mathrm{DR}(-\mathrm{T})$ ] with the first MR conditional single chamber pacemaker system [Evia SR $(-\mathrm{T})]$.

The purpose of our study was to evaluate feasibility of the new MR conditional pacemaker system (Evia SR-T and DR-T with Safio S53/S60 active screw-in leads, Biotronik SE \&Co KG, Berlin, Germany) under specific MR conditions. We report the results of the monocenter feasibility study of the MRI compatibility of the Evia pacemaker in combination with the Safio $S$ pacemaker lead.

\section{Methods}

From all patients implanted with permanent pacemakers at the general hospital of St. Pölten-Lilienfeld those patients implanted with Evia SR-T or DR-T PM and Safio $S$ leads at least 6 weeks before intended MR scan were eligible for enrollment in this single center prospective non-randomized pilot study. Pacemaker implantations were indicated according to current guidelines $[9,10]$. At enrollment pacing thresholds had to be $\leq 2.0$ Volts/0.4 ms, pacing impedances between 200 and 1.500 Ohms. The charge state of the battery had to be at least $30 \%$. Patients had to be afebrile. Patients must not have scheduled cardiac surgery within 3 months after enrollment. Patients gave written informed consent at enrollment. The study was approved by the local ethical committee and the Austrian competent authority.

\section{Evia pacemakers}

The Evia pacemakers (single chamber $[\mathrm{SR} / \mathrm{T}]$ and dual chamber $[\mathrm{DR} /-\mathrm{T}]$ ) are suited for all standard indications of bradycardia therapy. The contact surfaces of the pacemakers are titanium and epoxy resin. All pacemakers are rate-adaptive and multi-programmable. The Evia pacemaker offers atrial and ventricular capture control, ventricular pace suppression (VpS) algorithm and IEGM transmission via Home Monitoring (SR-T and DR-T only). For this study the Evia pacemakers (SR-T and DR-T) were used together with the Safio $S$ pacemaker leads. These pacemaker systems are conditionally MRI CE-approved. Safety and efficacy of the Evia pacemakers have been approved previously [11].

\section{Safio s leads}

The Safio $S$ is an active-fixation, transvenous, bipolar, endocardial lead designed for permanent atrial or ventricular stimulation and sensing. The lead is insulated with silicone, and has an IS-1 connector. The Safio S is flexible between the tip and ring electrodes and has an isodiametric design with a diameter of $6.6 \mathrm{Fr}$. The Safio $\mathrm{S}$ lead is a follow-up product of the Setrox $\mathrm{S}$ lead. The safety and efficacy of the Setrox lead was demonstrated earlier [12].

\section{Home monitoring}

The Evia DR-T and SR-T pacemakers have the ability to transmit and receive data over a distance of several meters using bi-directional long-range telemetry, i.e. without the need of a programming wand. The data is transmitted to a patient device (Cardio Messenger). Via mobile phone technique, the Cardio Messenger forwards the data to the Home Monitoring Service Center. Safety and efficacy of remote monitoring by Home Montoring in pacemaker patients have been demonstrated recently [13].

Within this study, the pacemaker based programmable Home Monitoring parameters were set as follows: automatic transmission time 2:00 am; periodical EGM transmission every 30 days; transmission in case of high atrial frequency and sustained atrial episode (Evia DR-T only); transmission in case of high ventricular frequency; kind of report: trend report.

\section{MR scans}

MR scans were conducted under consideration of previously published recommendations for MR in pacemaker patients [2,4-7]. After given written informed consent patients underwent a non-diagnostic MRI of the brain and the lower lumbar spine at 1.5 Tesla.

Before MR patients were asked for potential contraindications for MR scan. Tables 1 and 2 show in detail the MR scan protocols for the brain and for the lower lumbar spine, respectively. The brain scan consisted of 9 sequences with a total expected scan time of 14.5 minutes, and a dedicated receiving brain coil was used. Since MR scans were of a non diagnostic intention the last 2 sequences of the brain examination (CE-MRA and Perfusion) were performed without the administration of contrast agent. The lower lumbar spine scan consisted of 6 sequences and was expected to last 14.8 minutes. For the lower lumbar spine 
Sequences for Philips MRI Scanner

\begin{tabular}{|c|c|c|c|c|c|c|c|c|c|}
\hline & \multicolumn{9}{|c|}{ Body Region: Head, Patient Position: Supine, Patient Entry: Head First, Landmark on Eyes } \\
\hline & $\begin{array}{l}3 \text { plane } \\
\text { localizer }\end{array}$ & $\begin{array}{l}\text { Reference } \\
\text { Scan }\end{array}$ & AX SE T1 & AX FSE T2 & T2 FLAIR & Diffusion & 3D TOF MT & CE-MRA & Perfusion \\
\hline & $\begin{array}{l}\text { Landmark } \\
\text { on Eyes }\end{array}$ & $\begin{array}{l}\text { Landmark } \\
\text { on Eyes }\end{array}$ & $\begin{array}{l}\text { Landmark } \\
\text { on Eyes }\end{array}$ & $\begin{array}{l}\text { Landmark } \\
\text { on Eyes }\end{array}$ & $\begin{array}{l}\text { Landmark } \\
\text { on Eyes }\end{array}$ & $\begin{array}{l}\text { Landmark } \\
\text { on Eyes }\end{array}$ & $\begin{array}{l}\text { Landmark } \\
\text { on Eyes }\end{array}$ & $\begin{array}{l}\text { Landmark on } \\
\text { Eyes }\end{array}$ & $\begin{array}{c}\text { Landmark } \\
\text { on Eyes }\end{array}$ \\
\hline $\begin{array}{l}\text { Name Anatomy } \\
\text { Philips }\end{array}$ & $\begin{array}{l}\text { Head CNS- } \\
\text { Brain }\end{array}$ & Ref Scans & $\begin{array}{l}\text { Head CNS- } \\
\text { Brain }\end{array}$ & $\begin{array}{l}\text { Head CNS- } \\
\text { Brain }\end{array}$ & $\begin{array}{l}\text { Head CNS- } \\
\text { Brain }\end{array}$ & $\begin{array}{l}\text { Head CNS- } \\
\text { Function }\end{array}$ & $\begin{array}{l}\text { Head CNS- } \\
\text { Angio }\end{array}$ & $\begin{array}{l}\text { Head CNS-CE- } \\
\text { Angio }\end{array}$ & $\begin{array}{l}\text { Head CNS- } \\
\text { Function }\end{array}$ \\
\hline $\begin{array}{l}\text { Sequence Name } \\
\text { Philips }\end{array}$ & survey & RefScan & T1W_SE & T2W_TSE & T2W_FLAIR & DWI_E & S3DI_MC_HR & 3D_DYN_AVM & SPRESTO \\
\hline plane & ax/cor/sag & - & ax & ax & ax & $a x$ & $a x$ & sag & $a x$ \\
\hline Scan time & $0: 17$ & $0: 51$ & 02:39 & 02:13 & 03:18 & $00: 36$ & 02:52 & $00: 51$ & $0: 50$ \\
\hline stacks & 3 & 2 & 1 & 1 & 1 & 1 & 1 & 1 & 1 \\
\hline slices & 3 each & 82 & 18 & 18 & 16 & 18 & 100 & 10 & 30 \\
\hline Scan technique & T1-TFE & SENSE & SE & SE & $\mathbb{R}$ & SE & FFE & FFE & FFE \\
\hline $\begin{array}{l}\text { Fast imaging } \\
\text { mode }\end{array}$ & TFE & - & MS & TSE & TSE & EPI & none & none & EPI \\
\hline $\begin{array}{l}\text { (Turbo/EPI) } \\
\text { Factor }\end{array}$ & 42 & - & 1 & 19 & 23 & 89 & - & - & 19 \\
\hline $\mathrm{TE}$ [ms] & 5.2 & 0.95 & 15 & 100 & 120 & 104 & 6.9 & 1.27 & shortest \\
\hline $\mathrm{TR}[\mathrm{ms}]$ & 15 & 8 & 483 & 3607 & 6000 & 3034 & 24 & 5 & 22 \\
\hline $\mathrm{TI}[\mathrm{ms}]$ & - & - & - & - & 2000 & - & - & - & - \\
\hline Flip angle [deg] & 20 & 7 & 140 & 90 & 100 & 90 & 20 & 35 & 7 \\
\hline FOV $[\mathrm{mm}]$ & 250 & - & 230 & 230 & 230 & 230 & 200 & 260 & 220 \\
\hline Scan [\%] & 74 & 82 & 79 & 74 & 68 & 79.6 & 57.4 & 100 & 55.8 \\
\hline Scan matrix & $256 \times 126$ & $64 \times 52$ & $256 \times 163$ & 384 × 228 & $208 \times 115$ & $112 \times 89$ & $332 \times 190$ & $160 \times 144$ & $64 \times 29$ \\
\hline $\begin{array}{l}\text { Slice thickness } \\
{[\mathrm{mm}] \mathrm{acq} / \mathrm{rec}}\end{array}$ & 10 & - & 5 & 5 & 6 & 5 & $1 / 0.5$ & $16 / 8$ & 3.5 \\
\hline Slice gap & 0 & & 1 & 1 & 2 & 1 & - & - & - \\
\hline NSA / NEX & 1 & 3 & 2 & 3 & 2 & 1 & 1 & 1 & 1 \\
\hline $\begin{array}{l}\text { Bandwidth } \\
\text { [Hz/pixel] }\end{array}$ & 186.9 & 2540.7 & 109.3 & 224.0 & 152.9 & 1834 & 108.8 & 541.1 & 60.3 \\
\hline
\end{tabular}

scans an incorporated lumbar spine coil was used. Before performing the MR scans pacemakers were programmed to the MR mode (ProMRI feature). Within this feature the pacing mode was programmed according to the requirements of the patients to an asynchronous single chamber (A00 or V00), dual chamber mode (D00) or was deactivated (OFF). In case of programming an asynchronous pacing mode, the lower rate was automatically set to $80 \mathrm{bpm}$, and the output to 4.8 Volts $/ 1.0 \mathrm{~ms}$. Different pacemaker functions (e.g. capture control, memory functions) were deactivated during active ProMRI mode automatically. Upon programming the pacemaker in the ProMRI mode the Home Monitoring transmission system is automatically rendered inactive (i.e. OFF). Thus no risks of interferences between data transmission and MR system are to be expected.

There were certain restrictions to be considered for MR scans: since Evia pacemakers have an exclusion zone for the isocenter landmark (exclusion zone: below the level of the eyes and above the level of the major trochanters), the iso-center landmark had to be placed at the level of the eyes for brain scan, and at the level of the major trochanters for lumbar spine scan, respectively. The patient had to be in a supine position. No additional local sending inductors were allowed. The gradient slew rate had to be $\leq 200 \mathrm{~T} / \mathrm{m} / \mathrm{s}$. The whole body specific absorption rate (SAR) had to be $\leq 2 \mathrm{~W} / \mathrm{kg}$, whereas the head SAR had to be $\leq 3.2 \mathrm{~W} / \mathrm{kg}$, respectively. The scanning time per MR session should not exceed 30 minutes.

During MR patients were monitored using a MR compatible telemetry based ECG and pulse oximetry (In vivo Corporation Orlando, FL, USA). ECG and pulse oximetry curves were continuously observed by the first author and recorded on digital video tape for the purpose of (primarily not intended) retrospective evaluation. 
Table 2 MRI sequences for lumbar spine scan

Sequences for Philips MRI Scanner

\begin{tabular}{|c|c|c|c|c|c|c|}
\hline & \\
\hline & \multicolumn{6}{|c|}{ Body Region: Lumbar Spine, Patient Position: Supine, Patient Entry: Head First, Landmark on Trochanter } \\
\hline & Localizer & Sagittal T1 & Sagittal T2 & Axial T1 & Axial T2 & STIR \\
\hline & $\begin{array}{l}\text { Landmark on } \\
\text { Trochanter }\end{array}$ & $\begin{array}{l}\text { Landmark on } \\
\text { Trochanter }\end{array}$ & $\begin{array}{l}\text { Landmark on } \\
\text { Trochanter }\end{array}$ & $\begin{array}{l}\text { Landmark on } \\
\text { Trochanter }\end{array}$ & $\begin{array}{l}\text { Landmark on } \\
\text { Trochanter }\end{array}$ & $\begin{array}{l}\text { Landmark on } \\
\text { Trochanter }\end{array}$ \\
\hline $\begin{array}{l}\text { Name Anatomy } \\
\text { Philips }\end{array}$ & Spine-lumbar & Spine-lumbar & Spine-lumbar & Spine-lumbar & Spine-lumbar & Spine-lumbar \\
\hline $\begin{array}{l}\text { Sequence Name } \\
\text { Philips }\end{array}$ & survey & T1W_TSE_SAG & T2W_TSE_SAG & T1W_TSE(2)_TRA & T2W_TSE(2)_TRA & STIR_longTE_SAG \\
\hline plane & $\mathrm{ax} / \mathrm{cor} / \mathrm{sag}$ & sag & sag & $a x$ & $a x$ & sag \\
\hline Scan time [min] & $0: 28$ & 02:32 & $02: 12$ & 02:59 & 03:23 & 03:13 \\
\hline stacks & 3 & 1 & 1 & 3 & 3 & 1 \\
\hline slices & 3 each & 9 & 9 & 5 & 5 & 9 \\
\hline Scan technique & FFE & SE & SE & SE & SE & $\mathbb{R}$ \\
\hline $\begin{array}{l}\text { Fast imaging } \\
\text { mode }\end{array}$ & none & TSE & TSE & TSE & TSE & TSE \\
\hline $\begin{array}{l}\text { (EPI/Turbo) } \\
\text { Factor }\end{array}$ & & 5 & 21 & 4 & 18 & 17 \\
\hline TE [ms] & 3.9 & 8 & 120 & 8 & 120 & 80 \\
\hline $\mathrm{TR}$ [ms] & 23 & 380 & 2825 & 325 & 3500 & 3400 \\
\hline $\mathrm{TI}[\mathrm{ms}]$ & - & - & - & - & - & 165 \\
\hline Flip angle [deg] & 45 & 90 & 90 & 90 & 90 & - \\
\hline FOV $[\mathrm{mm}]$ & 400 & 160 & 160 & 200 & 200 & 160 \\
\hline Scan [\%] & 74.6 & 72.3 & 72,7 & 77.7 & 76 & 70.5 \\
\hline Scan matrix & $268 \times 200$ & $176 \times 240$ & $176 \times 241$ & $224 \times 174$ & $224 \times 171$ & $176 \times 238$ \\
\hline Slice thickness & 10 & 4 & 4 & 4 & 4 & 4 \\
\hline Slice gap & user defined & default & default & default & default & user defined \\
\hline NSA / NEX & 2 & 4 & 4 & 6 & 6 & 4 \\
\hline $\begin{array}{l}\text { Bandwidth [Hz/ } \\
\text { pixel] }\end{array}$ & 285.9 & 196.6 & 324,7 & 198.4 & 139.9 & 297.1 \\
\hline
\end{tabular}

Additionally patients were able to trigger manually an alarm in case of emergency. Emergency equipment was available onsite. After MR patients were asked for unusual perceptions (e.g. local heating, pacemaker vibration etc.) during MR scans.

\section{Primary study endpoints}

Primary endpoint \#1: MRI and pacing system related serious adverse device effect (SADE) free-rate

While all adverse events had to be recorded throughout the entire study, only the number of possibly pacing system and MRI related SADEs were the basis for endpoint calculation of the SADE rate. A SADE was pacing system related if it resulted from the presence or performance of the pacing system. A SADE was MR related if it occurred due to the interaction of the pacing system with the MR procedure. This was the case if the patient was within the 5 Gauss line of the MR system or if the SADE occurred in the month following the MR procedure. SADE's due to programming the pacemaker to $\mathrm{MR}$ mode were also classified as MR procedure related. Pocket and lead infections were described but not taken into account for the primary endpoint.

The parameter of interest PSADE was the SADE free rate per patient, which was calculated by $100 \%$ - (number of SADE divided by the number of patients)* $100 \%$. It was expected, that this SADE free rate will be greater than $90 \%$.

\section{Primary endpoint \#2: Pacing threshold rise (atrial and ventricular)}

The percentage of pacing leads with a pacing threshold rise between pre-MR follow-up (baseline FU) and 1month follow-up ( $\left.2^{\text {nd }} \mathrm{FU}\right)$ was investigated. The threshold behavior of the lead was defined as a success if the increase was not larger than or equal to 1.0 Volts. Only measurements of the same polarity (either uni- or bipolar) were taken into account. The proportion $\left(\mathrm{p}_{\mathrm{PT}}\right)$ of pacing threshold successes was calculated by dividing 
the number of leads without a pacing threshold rise as defined above by the total number of all leads.

\section{Secondary study end-points \\ $P$-wave sensing attenuation}

The P-wave sensing attenuation rate ( $\mathrm{p}_{\text {MRI Psensing }}$ ) was the percentage of patients who experienced a $\mathrm{P}$-wave sensing amplitude attenuation. P-wave amplitude attenuation was defined as either a P-wave amplitude decrease (between pre-MRI follow-up and 1-month follow-up) exceeding $50 \%$ or a $\mathrm{P}$-wave amplitude at 1-month follow-up smaller than $1.5 \mathrm{mV}$. In case that a patient fulfilled both conditions he/she was counted only once. The proportion ( $\mathrm{p}_{\text {MRI Psensing }}$ ) was calculated by dividing the number of patients with $\mathrm{P}$-wave sensing attenuation by the total number of patients. Only measurements of the same polarity (either uni- or bipolar) were taken into account. Patients with a P-wave sensing amplitude smaller or equal to $1.5 \mathrm{mV}$ at pre-MRI were excluded from the analysis of this endpoint.

For comparison: In the Setrox S Master Study the percentage of patients with a P-wave sensing amplitude lower than $1.5 \mathrm{mV}$ at the 3 -month follow-up was $4 / 109=3.7 \%$. In the same study, the percentage of patients with an amplitude decrease exceeding 50\% between 1-month and 3month follow-up was $1 / 97=1.0 \%$. This one patient had also a sensing amplitude below $1.5 \mathrm{mV}$ and therefore was not counted double. Hence a percentage ( $\mathrm{p}_{\text {Setrox }}$ Psensing $)$ of $3.7 \%$ resulted. For the purpose of this study the difference between the percentages were calculated via: $\Delta_{\mathrm{P} \text {-wave sensing }}=$ PMRI Psensing - psetrox Psensing and was expected to be zero or less.

\section{$R$-wave sensing attenuation}

The R-wave sensing attenuation rate ( $\mathrm{p}_{\text {MRI Rsensing }}$ ) was the percentage of patients who experienced a R-wave amplitude attenuation. R-wave amplitude attenuation was defined as either a R-wave amplitude decrease (between pre-MRI follow-up and 1-month follow-up) exceeding $50 \%$ or a R-wave amplitude at 1-month follow-up smaller than $5.0 \mathrm{mV}$. In case that a patient fulfilled both conditions he/she was counted only once. The proportion ( $\mathrm{p}_{\text {MRI Rsensing }}$ ) was calculated by dividing the number of patients with $\mathrm{R}$-wave sensing attenuation by the total number of patients. Only measurements of the same polarity (either uni- or bipolar) were taken into account. Patients with a R-wave sensing amplitude smaller or equal to $5.0 \mathrm{mV}$ at pre-MRI were excluded from the analysis of this endpoint.

For comparison: In the Setrox S Master Study the percentage of patients with a R-wave sensing amplitude lower than $5.0 \mathrm{mV}$ at the 3-month follow-up was 3/ $61=4.9 \%$ [8]. In the same study, the percentage of patients with a sensing decrease exceeding $50 \%$ between
1 -month and 3-month follow-up was $0 / 51=0.0 \%$. Therefore the percentage ( $p_{\text {Setrox Rsensing }}$ ) resulted to $4.9 \%$ [8].

For the purpose of this study the difference between the percentages was calculated via: $\Delta_{R \text {-wave sensing }}=p_{M R I}$ Rsensing PSetrox Rsensing and was expected to be zero or less.

\section{Follow-up}

Pacemakers were interrogated immediately before (baseline FU) and immediately after ( $\left.1^{\text {st }} \mathrm{FU}\right)$ MR to assess potential changes of lead parameters (right atrial (RA)/ right ventricular $(\mathrm{RV})$ sensing $[\mathrm{mV}]$, pacing threshold [V/0.4 ms], pacing impedance $[\mathrm{Ohm}]$ ) as well as of battery status $(100 \%$ or less). Patients were followed for

\section{Table 3 Patient demographics}

\begin{tabular}{|c|c|c|}
\hline & $\mathrm{N}$ & $\%$ \\
\hline Total patients & 30 & 100 \\
\hline Female & 12 & 40 \\
\hline Age (years) & \multicolumn{2}{|c|}{$73 \pm 12$} \\
\hline Height $(\mathrm{cm})$ & \multicolumn{2}{|c|}{$169 \pm 0.1$} \\
\hline Weight (kg) & \multicolumn{2}{|c|}{$80 \pm 16$} \\
\hline Body mass index & \multicolumn{2}{|c|}{$28 \pm 5$} \\
\hline \multicolumn{3}{|l|}{ Pacemaker indication } \\
\hline Higher degree AV block & 10 & 33 \\
\hline Sick sinus syndrome & 6 & 20 \\
\hline Brady-/Tachy-Syndrome & 7 & 23 \\
\hline AF with significant bradycardia & 7 & 23 \\
\hline AP (DC-PM only;\%; [median]) & \multicolumn{2}{|c|}{$34 \pm 33[27]$} \\
\hline VP (\%; [median]) & \multicolumn{2}{|c|}{$63 \pm 31[63]$} \\
\hline AF-Burden (DC-PM only;\%; [median]) & \multicolumn{2}{|c|}{$11 \pm 28[0]$} \\
\hline \multicolumn{3}{|l|}{ Pacing mode during MRI } \\
\hline $\mathrm{A} 00$ & 1 & 3 \\
\hline V00 & 3 & 10 \\
\hline \multirow[t]{2}{*}{ Do0OFF } & 5 & 17 \\
\hline & 21 & 70 \\
\hline \multicolumn{3}{|l|}{ Implanted pacemakers } \\
\hline Evia SR-T & 15 & 50 \\
\hline Evia DR-T & 15 & 50 \\
\hline \multicolumn{3}{|l|}{ Implanted leads } \\
\hline RA: Safio S $53 \mathrm{~cm}$ & 15 & 50 \\
\hline RV: Safio S $60 \mathrm{~cm}$ & 30 & 100 \\
\hline \multicolumn{3}{|l|}{ RV lead position } \\
\hline apical & 3 & 10 \\
\hline RVOT/septal & 27 & 90 \\
\hline Implantation site left pectorally & 30 & 100 \\
\hline PM pocket subcutaneously & 24 & 80 \\
\hline Time from implantation (months) & \multicolumn{2}{|c|}{$2.6 \pm 1.7$} \\
\hline
\end{tabular}


3 months with out-patient PM clinic visits at 1 month $\left(2^{\text {nd }} \mathrm{FU}\right)$ and 3 months $\left(3^{\text {rd }} \mathrm{FU}\right)$ after MR. Additionally, patients were remotely monitored using Home Monitoring based on routinely scheduled 30-day or event triggered transmissions.

\section{Statistical analyses}

Continuous variables are expressed as mean \pm SD. The effects of MR on sensing, pacing thresholds, and lead impedance were analysed by one-way ANOVA, for paired data followed by post-hoc analysis (Student's paired t-test). Categorical variables were compared using the chi-square test and the Fisher's exact test, where appropriate. Box plots display the following descriptive measurements: sample minimum: lower end of the whisker; Q1 (lower quartile): bottom of the box; Q2 (median): line inside the box; Q3 (upper quartile): top of the box; sample maximum: upper end of the whisker; mean: diamond inside the box; outliers: circles). A p-value $<0.05$ was considered significant.

\section{Results}

Thirty-one patients were enrolled. One male patient with an Evia DR-T had to be excluded because of an enrollment violation. The patient underwent scheduled surgery for single coronary bypass of the circumflex artery originating from the right coronary artery 4 weeks after the MR. The surgery was indicated before enrollment. Therefore,
30 patients are included in the following data analyses. Table 3 shows the characteristics of the patient cohort. The leading pacemaker indication was intermittent or permanent higher degree AV block ( $n=10$ [33\%]). Half of the patients had been implanted with dual chamber pacemakers. In a minority of patients $(n=6[20 \%])$ permanent pacemakers were placed in an intra- or sub pectoral pocket, mainly due to thin subcutaneous fatty tissue. The time from pacemaker implantation to enrollment/MR scan was $2.6 \pm 1.7$ months (median 1.7 months).

\section{MR scans}

Before MR all pacemakers were followed and were programmed according to the protocol. In all patients who were not pacemaker dependent $(n=21[70 \%])$ the pacing function was deactivated.

Tables 1 and 2 show the scan protocols for head and lower lumbar spine scan, respectively. Table 4 shows the mean values of SAR, scan duration and gradient strength for the different scan sequences. The total scan time of the brain scan was 14.0 minutes, of the lumbar spine scan 15.2 minutes, respectively.

In one patient, metallic artifacts surprisingly were recognized during the $1^{\text {st }}$ (survey) sequence of the head scan. MR was immediately stopped. After performing $\mathrm{x}$ ray demonstrating the metallic artifact (very small splinters) to be outside the osseous head and the decision that MR wouldn't cause any harm to the patient, the

Table 4 Measurements during MR scans

\begin{tabular}{|c|c|c|c|c|}
\hline Scan sequence & & SAR $(W / k g)$ & Scan duration (sec.) & Gradient strength (\% PNS) \\
\hline \multicolumn{5}{|c|}{ Head } \\
\hline 3 plane localizer & Head CNS-Brain & $0.3 \pm 0$ & $17.6 \pm 3.1$ & $16.0 \pm 0$ \\
\hline Reference Scan & Ref Scan & $0.3 \pm 0$ & $78.3 \pm 13.9$ & $19.5 \pm 1.4$ \\
\hline AX SE T1 & Head CNS-Brain & $2.0 \pm 0$ & $101.0 \pm 0$ & $12.1 \pm 1.6$ \\
\hline AX FSE T2 & Head CNS-Brain & $1.6 \pm 0$ & $133.0 \pm 0$ & $36.8 \pm 0.6$ \\
\hline T2 Flair & Head CNS-Brain & $0.5 \pm 0$ & $198.0 \pm 0$ & $30.5 \pm 0.6$ \\
\hline Diffusion & Head CNS-Function & $0.2 \pm 0$ & $36.0 \pm 0$ & $52.7 \pm 1.2$ \\
\hline 3D TOF MT & Head CNS-Angio & $1.8 \pm 0$ & $169.0 \pm 0$ & $35.9 \pm 4.7$ \\
\hline CE-MRA & Head CNS-CE-Angio & $1.9 \pm 0.2$ & $56.0 \pm 0$ & $14.0 \pm 0$ \\
\hline Perfusion & Head CNS-Angio & $0.2 \pm 0.9$ & $51.0 \pm 0$ & $51.2 \pm 9.7$ \\
\hline Total scan time (min) & & & 14.0 & \\
\hline \multicolumn{5}{|c|}{ Lumbar spine } \\
\hline Localizer center at L1 & Survey & $0.3 \pm 0$ & $53.0 \pm 0$ & $17.0 \pm 0$ \\
\hline Sagital T1 Isocenter at L1 & T1W_TSE_SAG & $2.0 \pm 0$ & $158.0 \pm 0$ & $38.7 \pm 1.3$ \\
\hline Sagital T2 Isocenter at L1 & T2W_TSE_SAG & $2.0 \pm 0$ & $132.0 \pm 0$ & $40.0 \pm 0$ \\
\hline Axial T1 Isocenter at L1 & T1W_TSE (2)_TRA & $2.0 \pm 0$ & $179.0 \pm 0$ & $40.0 \pm 0$ \\
\hline Axial T2 Isocenter at L1 & T2W_TSE (2)_TRA & $1.6 \pm 0$ & $203.0 \pm 0$ & $39.6 \pm 2.0$ \\
\hline Sagital T1 Isocenter S1 & STIR_long TE_SAG & $1.9 \pm 0$ & $193.0 \pm 0$ & $41.0 \pm 0$ \\
\hline Total scan time (min) & & & 15.2 & \\
\hline
\end{tabular}


survey sequence was started again and the whole protocol was completed. The metallic splinters were unknown even to the patient and might be the result from working with an angle grinder.

None of the patients reported about uncomfortable feelings or other disorders during MR possibly related to the pacemaker.

MR scans were performed in a non-diagnostic manner, but were analyzable concerning rough conspicuousnesses. Analyses of MR scans revealed no important incidental findings.

After completion of all MR sequences pacemakers were interrogated again and re-programmed. Total time from activation to deactivation of the ProMRI feature was $73 \pm 17$ minutes (median 74).

\section{Adverse events}

\section{$M R I$ related adverse events}

No MRI related early or late adverse events occurred. Therefore, the $1^{\text {st }}$ primary endpoint was met $\left(\mathrm{p}_{\mathrm{SADE}}=100 \%\right)$.

\section{Other adverse events}

There were 4 AEs. All AEs were not MR related. One female patient (age 56 years, bradycardia/tachycardia syndrome, normal left ventricular function (LVF) prior to PM implantation, VVI-PM [apical lead position]) suffered twice from cardiac decomposition $\left(1^{\text {st }}\right.$ episode 43 days after MR, $2^{\text {nd }}$ episode 66 days after MR) presumably due to the effects of frequent pacing from the right ventricular apex (ventricular pacing: $61 \%$ at baseline FU, $70 \%$ at $2^{\text {nd }} \mathrm{FU}$ ) as well as of intermittent rapidly conducted atrial fibrillation. Echocardiography revealed a significantly reduced LVF at the time of first cardiac decomposition. Another female patient (age 83 years, higher degree AV block, DR-PM) was admitted 11 days after MR to our emergency department due to suspected deep vein thrombosis of the left lower leg (which was excluded). One male patient (age 85 years, coronary artery disease, status post $\mathrm{CABG}$, persistent atrial fibrillation, VVI-PM) died suddenly 17 days after MR. The patient was admitted to our emergency department 10 days after the MR scan because of newly recognized icterus. During the in-hospital stay pancreatic carcinoma with disseminations was diagnosed. On $14^{\text {th }}$ of August, 2 hours after discussion about prognosis of the malignant disease and potential further diagnostic and therapeutic options the patient died suddenly. Autopsy revealed a large myocardial infarction as underlying cause of death. Post mortem PM interrogation showed fast VT at the time of death (Figure 1).

\section{Lead measurements}

Since one patient died before passing the 1-month FU, calculation of the study endpoints is based on the data of 29 patients. The 1-month follow-up took place after a mean of 1.1 months after the MR procedure, the 3month follow-up after 3.0 months, respectively.

All lead measurements were done in a bipolar configuration. Except for RV pacing impedance assessed immediately after the MR paired Student's t-test revealed no significant differences of lead dependent parameters as well as of battery status between the pre-MRI FU and all other FUs. Table 5 gives an overview of all measurements from all available FUs.

\section{Atrial pacing threshold}

Atrial pacing thresholds showed a slight increase in the course after the MRI scans (Figure 2a). The measured values were not significantly different between the follow-ups (Table 5). One patient had an increase of the atrial pacing threshold by $75 \%$ (from $0.4 \mathrm{~V} / 0.4 \mathrm{~ms}$ to $0.7 \mathrm{~V} / 0.4 \mathrm{~ms}$ ) from the $1^{\text {st }} \mathrm{FU}$ to the $2^{\text {nd }} \mathrm{FU}$. There was no further change observed until the 1-month FU.

\section{Ventricular pacing threshold}

Ventricular pacing thresholds showed a slight increase between the 1-month FU and the 3-month FU (Figure 2b),

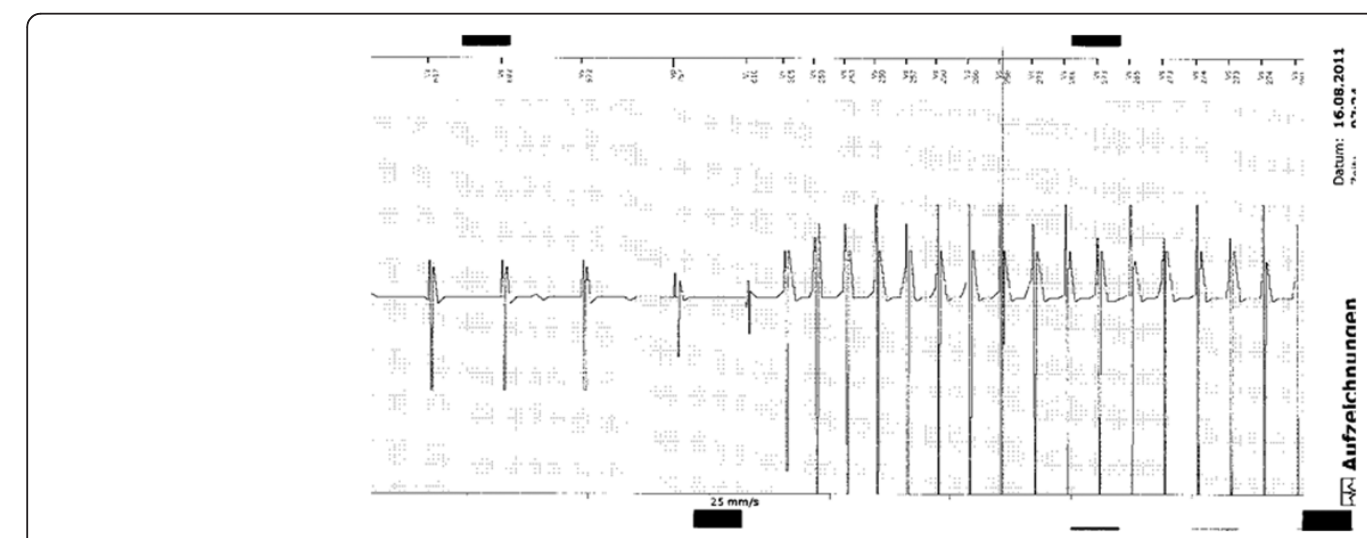

Figure 1 Patient \#31. Episode print-out of spontaneously occurring VT at the time of myocardial infarction/death. 
Table 5 Lead Measurements and Battery status

\begin{tabular}{|c|c|c|c|c|c|}
\hline \multirow[b]{2}{*}{ Parameter } & \multicolumn{5}{|c|}{ Follow-up } \\
\hline & $1^{\text {st }} \mathrm{FU}(\mathrm{n}=30)$ & $2^{\text {nd }} \mathrm{FU}(\mathrm{n}=30)$ & $3^{\text {rd }} \mathrm{FU}(n=29)$ & $4^{\text {th }} \mathrm{FU}(\mathrm{n}=29)$ & $p=*$ \\
\hline Time from MRI (months) & - & - & $1.1 \pm 0.3$ & $3.0 \pm 0.3$ & \\
\hline RA sensing $(\mathrm{mV})$ & $3.2 \pm 2.1$ & $3.2 \pm 2.3$ & $3.2 \pm 2.1$ & $3.1 \pm 2.0$ & n.s. \\
\hline RA pacing threshold (V@0,4 ms) & $0.68 \pm 0.18$ & $0.67 \pm 0.16$ & $0.71 \pm 0.24$ & $0.73 \pm 0.15$ & n.s. \\
\hline RA pacing impedance (Ohms) & $507 \pm 55$ & $500 \pm 46$ & $520 \pm 50$ & $495 \pm 59$ & n.s. \\
\hline RV sensing $(m V)$ & $15.0 \pm 6.0$ & $15.0 \pm 6.0$ & $14.9 \pm 6.5$ & $14.7 \pm 6.3$ & n.s. \\
\hline RV pacing threshold (V@0,4 ms) & $0.78 \pm 0.22$ & $0.79 \pm 0.20$ & $0.78 \pm 0.22$ & $0.82 \pm 0.24$ & n.s. \\
\hline RV pacing impedance (Ohms) & $608 \pm 54$ & $599 \pm 52^{+}$ & $607 \pm 47$ & $597 \pm 57$ & n.s. \\
\hline Battery status (\%) & $100 \pm 0$ & $100 \pm 0$ & $100 \pm 0$ & $99.7 \pm 1.3$ & n.s. \\
\hline \multicolumn{6}{|c|}{ Value changes (\% [median]; compared with pre MRI FU) } \\
\hline RA sensing & & $-2 \pm 20[0]$ & $+6 \pm 43[0]$ & $+15 \pm 57[8]$ & \\
\hline RA PTH & & $+2 \pm 23[0]$ & $+2 \pm 30[0]$ & $+9 \pm 7[0]$ & \\
\hline RA Pimp & & $-1 \pm 4[0]$ & $+3 \pm 6[4]$ & $-2 \pm 11[0]$ & \\
\hline RV sensing & & $-1 \pm 6[-1]$ & $-2 \pm 12[-2]$ & $-4 \pm 14[-1]$ & \\
\hline RV PTH & & $+2 \pm 8[0]$ & $0 \pm 12[0]$ & $7 \pm 24[0]$ & \\
\hline RV PImp & & $-2 \pm 2[-2]$ & $0 \pm 6[0]$ & $-2 \pm 7[0]$ & \\
\hline Battery status & & $0 \pm 0[0]$ & $0 \pm 0[0]$ & $0 \pm 1[0]$ & \\
\hline
\end{tabular}

Grey columns: relevant FUs for endpoint calculation.

*Oneway ANOVA.

$+p<0.05$ when comparing with pre MRI values (paired t-test).

but the measured differences were not significantly different (Table 5).

The female patient which decompensated twice showed an increase of the ventricular PTH from $0.8 \mathrm{~V} /$ $0.4 \mathrm{~ms}$ at the 1 -month $\mathrm{FU}$ to $1.5 \mathrm{~V} / 0.4 \mathrm{~ms}$ at the 3month FU (+88\%) most likely due to new rhythm control therapy with amiodarone.

All other atrial and ventricular pacing threshold changes observed were less than $50 \%$. None of the patients had an increase of the atrial or ventricular PTH by 1 Volt or more between the baseline FU and the 1month FU. Therefore, the second primary endpoint was met $\left(\mathrm{p}_{\mathrm{PT}}=1\right)$.

\section{Atrial sensing}

Values for atrial sensing were stable throughout the study period (Table 5, Figure 2c). Three patients had an atrial sensing of $\leq 1.5 \mathrm{mV}$ at the pre-MRI FU and therefore - were excluded from calculation of the first secondary endpoint. Since no one of the remaining patients $(n=12)$ had an atrial sensing of $<1.5 \mathrm{mV}$ or had a sensing attenuation by $\geq 50 \%$, also the first secondary endpoint was met ( $\mathrm{p}_{\text {MRI Psensing }}=0.0 \%$ ). $\Delta_{\mathrm{P} \text {-wave sensing }}$ was calculated to be $-3.7 \%$.

\section{Ventricular sensing}

Values for ventricular sensing were stable throughout the study period (Table 5, Figure 2d). Two patients had no intrinsic $\mathrm{R}$ wave (one at the pre-MRI FU, one at the 1-month FU), one patient died before the 1-month-FU. These 3 Patients were excluded from calculation of the second secondary endpoint. Since no one of the remaining patients $(\mathrm{n}=27)$ had a ventricular sensing of $<5.0 \mathrm{mV}$ nor had a sensing attenuation by $\geq 50 \%$, also the $2^{\text {nd }}$ secondary endpoint was met ( $\mathrm{p}_{\text {MRI Rsensing }}=$

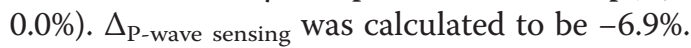

\section{Home monitoring}

Within the observational period more than 1800 data sets were transmitted, composed of - amongst other parameters - RA/RV Impedance, RA/RV Threshold, RA/RV Sample Amplitude, RA/RV Sample Amplitude Mean, Heart Rate $(24 \mathrm{~h})$ and Battery Status. Neither hardware related annotations (e.g. pacing impedance alert, battery depletion) were transmitted nor device related (hardware/software) irregularities were found in the status information based on routinely scheduled 30day or event triggered transmissions.

\section{Pacemaker associated MR imaging artifacts}

In all patients the presence of the pacemaker caused an inhomogeneity of the static magnetic field in the mid face and the frontal lobe areas resulting in a MR imaging artifact on diffusion weighted sequences of the brain (Figure 3). 


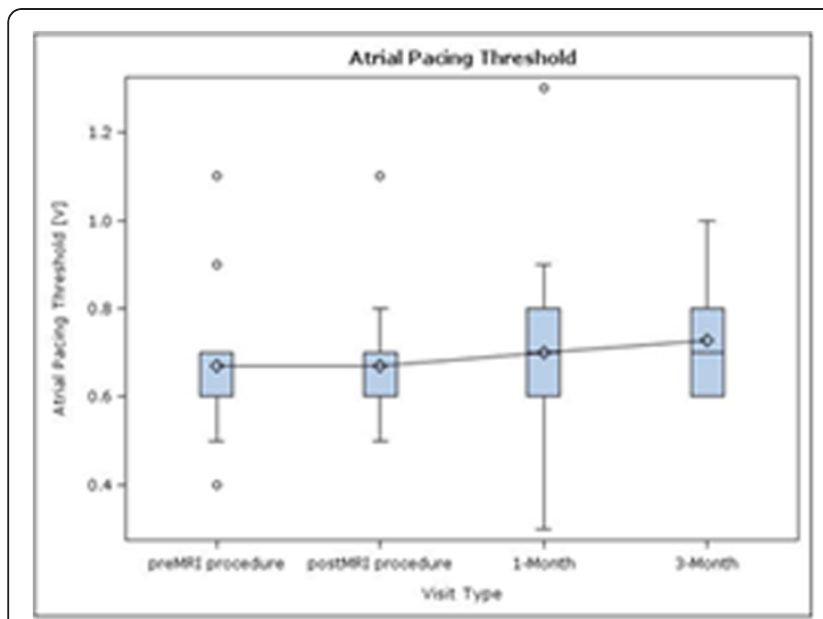

a.

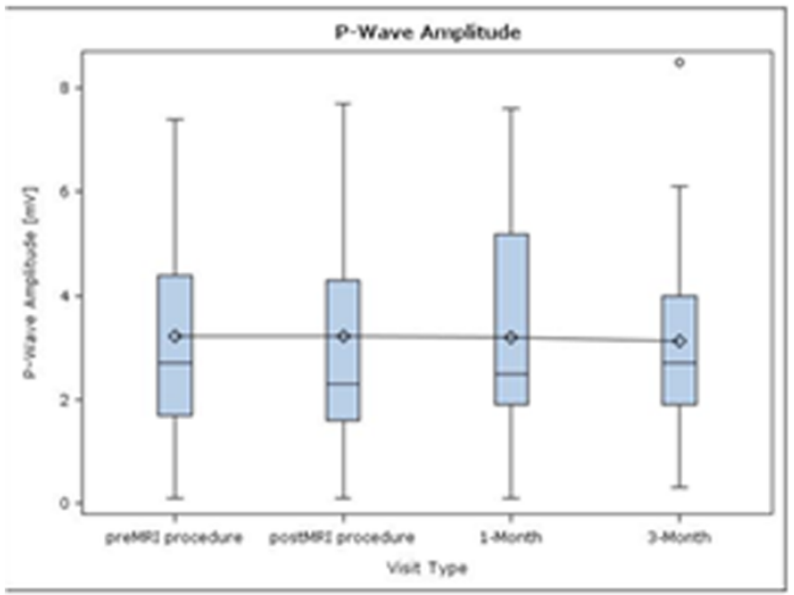

C.

Figure 2 The diagrams show the changes of RA-PTH (a), RV-PTH (b), P wave sensing amplitude (c) and R-wave sensing amplitude (d) at the different FUs.

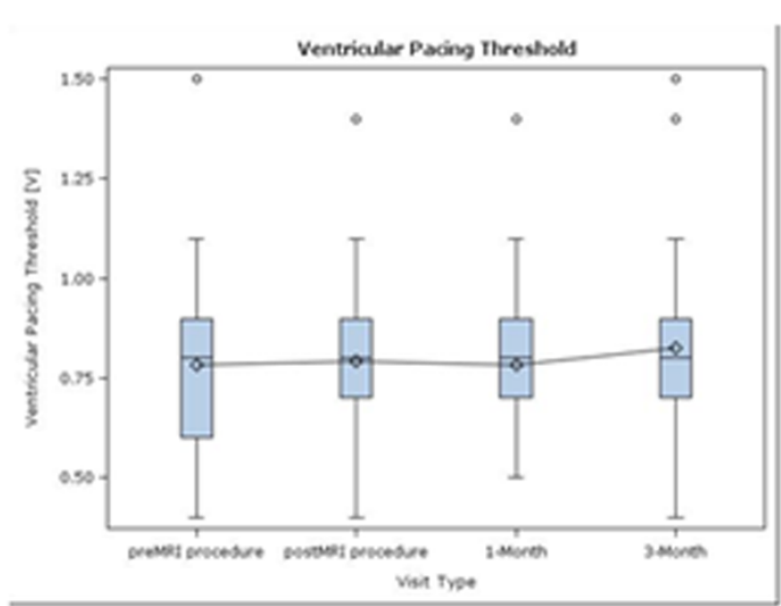

b.

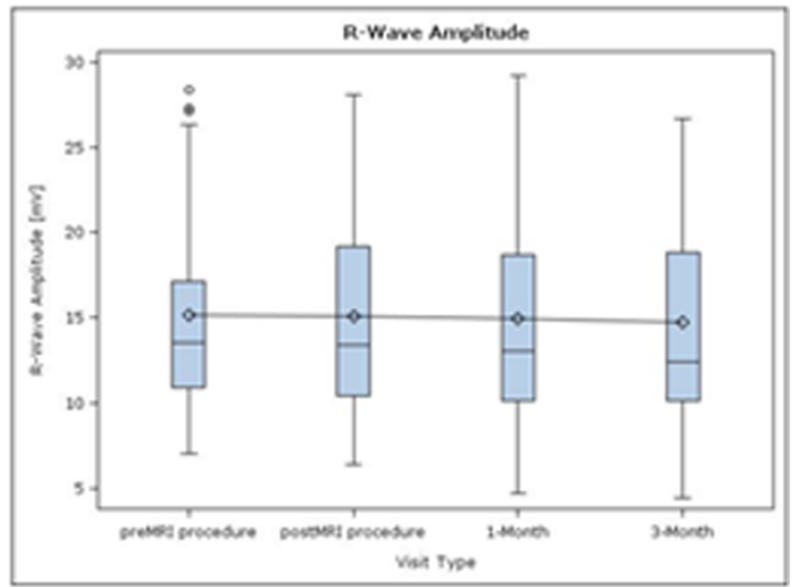

d.

\section{Discussion}

This study is the first to report about functionality of the MR conditional Evia pacemaker in a MR environment, and the second study that reports about MR eligible pacemaker systems undergoing MR [8].

The MR conditional Evia pacemakers with Safio S leads demonstrated unobtrusive function under MR conditions. The use of the Evia pacemakers with Safio $\mathrm{S}$ leads in MR environment was feasible.

Since there were no MR related SADEs nor were significant pacing threshold increases or sensing attenuations as defined by the protocol all primary and secondary study endpoints were met. Lead measurements were not affected by MR immediately nor showed significant changes within the observational period of 3 months.

The continuous observation of the ECG and pulseoxy monitor showed no inhibition of pacemaker stimulation, sustained or non-sustained atrial and/or ventricular arrhythmias, asystole, or other unexpected changes of heart rate occurred in the context of MR scans. Also no conspicuousnesses concerning pacemaker statistics and the functions or the electrical integrity of the devices occurred during or after MR. No patient reported unusual feelings/sensations that potentially were related to the pacemaker while being in the MR environment. Observed differences in lead measurements between the different follow-ups were in clinically accepted ranges.

The observed pacemaker associated MR imaging artifact of the brain on diffusion weighted images (Figure 3) may have no relevant clinical importance, since the image quality of the remaining sequences such as FLAIR and T2 weighted spin-echo images is not affected (Figure 3).

As recommended patient surveillance during the MRI scans was realized by using a combination of ECG and pulse oximetry [2-7]. Verbal contact with the patient was nearly impossible especially during the brain scan. 


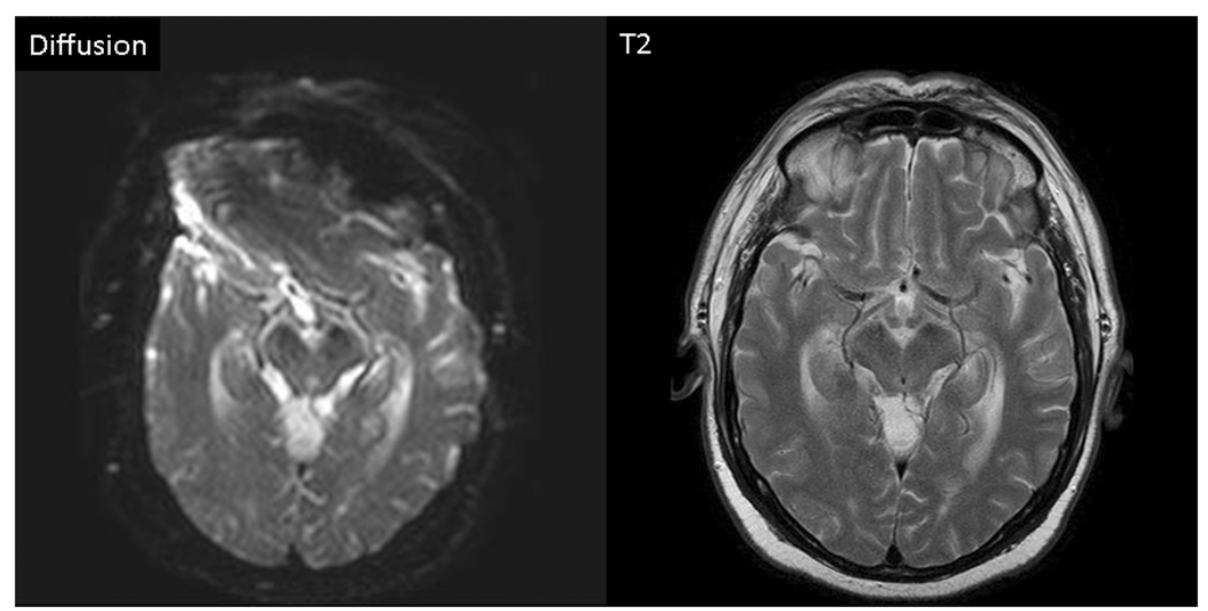

Figure 3 Left: Diffusion weighted gradient echo image. Notice pacemaker associated distortion of the image in the frontal region of the brain. Right: $\mathbf{T} 2$ weighted spin echo image (identical slice location) showing normal anatomy not affected by the presence of a pacemaker.

ECG is sometimes highly affected by radio frequencies during MR scans and only useable in-between the MR sequences. Our experience is that only pulse oximetry in conjunction with a patient activated alert system seems to be reliable for patient surveillance during total scan time, whereas ECG with appropriate quality is reliable only occasionally during the scans.

Another finding of the study is that patients were programmed to the ProMRI mode for a mean of $73 \pm 17$ minutes. This fact has impact on patient surveillance not only during MR scan, but also before and thereafter. This long period observed in our study was a result from cumulative 30 min MR scans, changing the body position in relation to the MR bore between the two different scans, taking off clothes before and on thereafter, and placement of the technical equipment for the purpose of patient surveillance. Additional time was needed for awaiting availability of the MR scanner since study scans were performed within daily routine of the private radiology institute. Although the pacing function was set in all non-pacemaker dependent patients $(\mathrm{n}=21[70 \%])$ to OFF when we programmed the pacemakers into the ProMRI mode, the long period our patients remained in the ProMRI mode had no impact on patient safety within our study since pacemaker programming was done in or near the control room of the MR scanner, and patients never were on their own. But what to do in the case pacemaker programming is located far away from the MR scanner (e.g. out-patient pacemaker office and radiology department located in different facilities). In this case, programming the pacemaker off may provoke complications like syncope on the way to or back from the scanner. This scenario may be unlikely in this low-risk patient group, but would have legal implications if it happens. There are several ways to overcome this problem. The personnel intensive way is to guide the patient e.g. from the pacemaker out-patient clinic to the MR scanner and later back again. Another way is to remotely activate/deactivate the specific MR mode just when the patient enters/leaves the room where the MR scanner is located. Of course, one could abstain from programming a different than an asynchronous pacing mode. Indeed asynchronous pacing was judged to be safe, but only for a short time as it usually is used during pacemaker follow-up [14]. Seen from this point of view there was (and would have been) long term asynchronous pacing in our cases. This fact may be another source of hidden danger when performing MR even in patients with MR conditional pacemakers and all those who are involved should be aware of this [15].

\section{Limitations}

The major limitation of our study is the limited number of patients and the non-randomized and - therefore statistically not powered study design. However, the intention of the study was to collect experience in order to prepare the ProMRI AFFIRM master study of the MRI compatibility of the Evia/Entovis pacemaker in combination with Safio S leads.

\section{Conclusion}

The new MR conditional Evia pacemaker system demonstrated unobtrusive function under MR conditions. Observed differences in lead measurements between the different follow-ups were in clinically accepted ranges. No MR related adverse events occurred. Pacemaker related MR imaging artifacts occurred on diffusion weighted sequences of the brain, but may have no clinical relevance.

Our study demonstrated feasibility and safety of Evia single chamber and dual chamber pacemakers with Safio $S$ leads in a MRI environment under well defined 
conditions. The long duration patients remained in the specific MR pacemaker programming mode (ProMRI) may have impact on patient safety and, therefore - will influence working algorithms for safe performance of $\mathrm{MR}$ in patients even with MR conditional pacemaker systems.

\section{Abbreviations}

AE: Adverse event; CABG: Coronar artery bypass graft(ing); CE: European conformity; DR: Dual chamber rate responsive; ECG: Electrocardiogram; Fr: French; FU: Follow-up; IEGM: Intracardiac electrogram; Kg: Kilogramms; MR: Magnetic resonance; MRI: Magnetic resonance imaging; Ms: Millisecond (s); mV: Millivolt(s); PM: Pacemaker(s); PTH: Pacing threshold; RF: Radio frequency; RA: Right atrial; RV: Right ventricular; RVOT: Right ventricular outflow tract; SADE: System related serious adverse device effect; SAR: Specific absorption rate; SD: Standard deviation; SR: Single chamber rate responsive; V: Volt(s); VP: Ventricular pacing; VT: Ventricular tachycardia; W: Watt(s)

\section{Competing interests}

1 Biotronik (lecture fees, travel grants), Boston Scientific (lecture fees, travel grants), Medtronic (travel grants), St. Jude Medical (lecture fees, travel grants). ${ }^{2}$ None.

${ }^{3}$ Biotronik (lecture fees, travel grants), Boston Scientific (lecture fees, travel grants), Medtronic (lecture fees, travel grants), St. Jude Medical (lecture fees, travel grants).

${ }^{4}$ Biotronik employee.

${ }^{5}$ Medtronic (lecture fees, travel grants).

\section{Authors' contributions}

CGW substantial contributions to conception and design; acquisition of data, and analysis and interpretation of data; drafting the manuscript; final approval of the version to be published. ES substantial contributions to conception and design; interpretation of data; drafting the manuscript; final approval of the version to be published. PV revising it critically for important intellectual content; final approval of the version to be published. BN substantial contributions to conception and design; acquisition of data, and analysis and interpretation of data; revising manuscript critically for important intellectual content; final approval of the version to be published. HM substantial contributions to conception and design; revising manuscript critically for important intellectual content; final approval of the version to be published. All authors read and approved the final manuscript.

\section{Acknowledgments}

We thank Menges Medical (Gallspach, Austria; www.memed.at) for their support by providing the study with the telemetry based MR conditional monitoring device.

We thank Michael Griesmayer (radiology technician) for his outstanding engagement and support of MR scans.

The study was funded by Biotronik SE\&Co KG., Berlin, Germany

\section{Author details}

'Hospital of St. Pölten-Lilienfeld, Department of Cardiology, St. Pölten, Austria. ${ }^{2}$ Institute of Diagnostic Imaging, Frühwald, Steiner, Obermayer, St. Pölten, Austria. ${ }^{3}$ Karl Landsteiner Society, Institute of Research on Ischemic Heart Diseases and Rhythmology, St. Pölten, Austria. ${ }^{4}$ Biotronik SE \& Co KG, Berlin, Germany.

Received: 15 March 2012 Accepted: 17 August 2012

Published: 25 September 2012

\section{References}

1. American College of Radiology: ACR Practice Guidelines. http://www.acr.org.

2. Roguin A, Schwitter J, Vahlhaus C, Lombardi M, Brugada J, Vardas P, Auricchio A, Priori S, Sommer T: Magnetic resonance imaging in individuals with cardiovascular implantable electronic devices. Europace 2008, 10:336-346.

3. Kalin R, Stanton MS: Current clinical issues for MRI scanning of pacemaker and defibrillator patients. PACE 2005, 28:326-328.

4. Sommer T, Naehle CP, Yang A, Zeijlemaker V, Hackenbroch M, Schmiedel A, Meyer C, Strach K, Skowasch D, Vahlhaus C, Litt H, Schild H: Strategy for safe performance of extrathoracic magnetic resonance imaging at 1.5 Tesla in the presence of cardiac pacemakers in non-pacemaker dependent patients. A prospective study with 115 examinations. Circulation 2006, 114:1285-1292.

5. Nazarian S, Roguin A, Zviman MM, Lardo AC, Dickfeld TL, Calkins H, Weiss RG, Berger RD, Bluemke DA, Halperin HR: Clinicial utility of a protocol for noncardiac and cardiac magnetic resonance imaging of patients with permanent pacemakers and implantable-cardioverter defibrillators at 1.5 Tesla. Circulation 2006, 114:1277-1284.

6. Martin ET, Coman JA, Shellock FG, Pulling CC, Fair R, Jenkins K: Magnetic resonance imaging and cardiac pacemaker safety at 1.5-Tesla. Journal of the American College of Cardiology 2004, 43:1315-1324.

7. Levine GN, Gomes AS, Arai AE, Bluemke DA, Flamm SD, Kanal E, Manning WJ, Martin ET, Smith JM, Wilke N, Shellock FS: Safety of magnetic resonance imaging in patients with cardiovascular devices. An American heart association scientific statement from the committee on diagnostic and interventional cardiac catheterization, council on clinical cardiology, and the council on cardiovascular radiology and intervention. Circulation 2007, 116:2878-2891.

8. Wilkoff BL, Taborsky M, Vymazal J, Kanal Heuer H, Hecking K, Johnson WB, Young W, Ramza B, Akhtar N, Kuepper B, Hunold P, Luechinger R, Puererfellner H, Duru F, Gotte MJ, Sutton R, Sommer T, EnRhythm MRI SureScan Pacing System Study Investigators: Magnetic resonance imaging in patients with a pacemaker system designed for the magnetic resonance environment. Heart Rhythm 2011, 8:65-73.

9. Vardas PE, Auricchio A, Blanc J-J, Daubert J-C, Drexler H, Ector H, Gasparini M, Linde C, Morgado FB, Oto A, Sutton R, Trusz-Gluza M, European Society of Cardiology; European Heart Rhythm Association: Guidelines for cardiac pacing and cardiac resynchronization therapy. The task force for cardiac pacing and cardiac resynchronization therapy of the European society of cardiology in collaboration with the European heart rhythm association. European Heart J 2007, 28:2256-2295.

10. Epstein AE, DiMarco JP, Ellenbogen KA, Estes NA 3rd, Freedman RA, Gettes LS, Gillinov AM, Gregoratos G, Hammill SC, Hayes DL, Hlatky MA, Newby LK, Page RL, Schoenfeld MH, Silka MJ, Stevenson LW, Sweeney MO, Smith SC Jr, Jacobs AK, Adams CD, Anderson JL, Buller CE, Creager MA, Ettinger SM, Faxon DP, Halperin JL, Hiratzka LF, Hunt SA, Krumholz HM, Kushner FG, et al.: American college of cardiology/American heart association task force on practice guidelines. ACC/AHA/HRS 2008 guidelines for devicebased therapy of cardiac rhythm abnormalities: a report of the American college of cardiology/American heart association task force on practice guidelines (writing committee to revise the ACC/AHA/NASPE 2002 guideline update for implantation of cardiac pacemakers and antiarrhythmia devices) developed in collaboration with the American association for thoracic surgery and society of thoracic surgeons. J Am College of Cardiology 2008, 51 21:e1-e62.

11. Final report for the master study of the Evia DR-T/DR/SR-T/SR pacemaker. 2010, BIOTRONIK GmbH \& Co KG. [Biotronik internal 2010].

12. PZK-Clinical Report 29-1, October 24, 2006., BIOTRONIK GmbH \& Co KG. [Biotronik internal 2006].

13. Mabo P, Victor F, Bazin P, Ahres S, Babuty D, Da Costa A, Binet D, Daubert $\mathrm{JC}$, on behalf of the COMPAS trial Investigators: A randomized trial of longterm remote monitoring of pacemaker recipients (The COMPAS trial). European Heart J 2011, Epub ahead of print.

14. Nowak B, Hemmer W, Israel CW, Krämer LI, Neuzner J, Pfeiffer D, Rybak K Schuchert A, Wiegand UKH: Stellungnahme der Arbeitsgruppe Herzschrittmacher der Deutschen Gesellschaft für Kardiologie zur Sicherheit der asynchronen ventrikuläre Schrittmacherstimulation Herausgegeben vom Vorstand der Deutschen Gesellschaft für Kardiologie - Herz- und Kreislaufforschung e.V. Bearbeitet im Auftrag der Kommission für klinische Kardiologie. Clinical Research in Cardiology 2006, 95:57-60.

15. Colletti PM, Shinbane JS, Shellock FG: "MR-conditional" pacemakers: the radiologist's role in multidisciplinary management. Am J Radiology 2011, 197:W457-W459.

\section{doi:10.1186/1532-429X-14-67}

Cite this article as: Wollmann et al:: Monocenter feasibility study of the MRI compatibility of the Evia pacemaker in combination with Safio S pacemaker lead. Journal of Cardiovascular Magnetic Resonance 2012 14:67. 